\title{
Dermatoosteolysis, Kirghizian type
}

INSERM

\section{Source}

INSERM. (1999). Orphanet: an online rare disease and orphan drug data base.

Dermatoosteolysis, Kirghizian type. ORPHA:1657

Dermatoosteolysis, Kirghizian type, is characterised by recurrent skin ulceration,

arthralgia, fever, peri-articular osteolysis, oligodontia and nail dystrophy. This disease has been described in five sibs in a family of Kirghizian orig in (Central Asia). Three of the sibs also presented with keratitis leading to visual impairment or blindess. Transmission is autosomal recessive. 